\title{
L'enlèvement : une arme centrale de la Guerre du Liban (1975-1990)
}

\author{
Chloé Rabanes \\ (Doctorante au Centre d'études historiques - \\ EHESS)
}

\section{Résumé/Abstract}

[Fr] L'enlèvement est une forme originale de violence dont la compréhension est contrainte par la visibilité exclusive de certaines de ses pratiques, notamment les prises d'otages et les disparitions. À ce titre, elle a toujours été exclue des univers guerriers et n'a jamais été considérée comme une modalité de combat. Pourtant, les dizaines de milliers d'enlèvements qui ont lieu au Liban de 1975 à 1990 témoignent de la centralité d'une telle pratique dans un conflit civil où s'imbriquent des logiques complexes. Massivement mobilisée par les combattants, la capture s'est imposée comme une arme incontournable du répertoire d'action guerrier et son étude permet d'interroger les mécanismes à l'œuvre sur le terrain. Violence adaptée aux rythmes de la guerre et aux configurations particulières des combats, l'enlèvement est mis au service d'objectifs multiples. Moyen d'échange et de négociation avec l'ennemi, rituel de vengeance, instrument d'homogénéisation des espaces et de contrôle des territoires, les captures disent beaucoup des enjeux stratégiques de la guerre et du rapport à l'ennemi. Utilisés de façon permanente durant les quinze années de conflit et touchant indistinctement civils et militaires, les enlèvements ont constitué une des formes les plus anxiogènes de la guerre et un traumatisme durable pour la population. Imprévisible et brutale, la détention des corps pose des enjeux inédits en termes de violence infligée et subie qui permettent de pénétrer au cœur d'une réalité longtemps oubliée : celle de l'expérience de guerre libanaise. Cette «nouvelle» arme interroge également les formes du combat moderne. La multiplicité de ses usages et les rapports équivoques à l'ennemi qu'elle exprime expliquent peut-être qu'elle se soit faite une place importante dans un conflit aux configurations peu classiques.

Mots clés : Liban, guerre civile, enlèvement, capture, violence.

[En] Abduction is an original form of violence generally understood as limited to two of its most visible forms : kidnapping and disappearances. As a result, abduction is rarely taken into account in the universe of war and is not considered as a mode of combat. In spite of this, the tens of thousands of abductions that took place in Lebanon between 1975 and 1990 illustrate the importance of this practice in the context of complex armed civil conflicts. Due to its widespread use by combatants, abduc- 
tion became one of the central means by which war was fought. Analysing abduction allows us to shed light on the mechanisms used on the ground during this period of war. Abduction was used as a means of achieving several objectives, thanks in part to its capacity to adapt to the rhythm of war and the different forms combat may take. Abductions illustrate some of the strategic issues of war and the nature of the relationship between rivals. They act as a means of negotiating with the opponent and they also represent a ritual of vengeance and a means of shifting the balance of spatial control. As a result of their permanent use during fifteen years of conflict and their effects on both the civilian and military populations, abductions constitute one of the most traumatising aspects of war, producing long term-effects on inhabitants. The withholding of individuals as an act of war raises a series of unanswered questions that open the door to a long-forgotten reality : that of the Lebanese war experience. This "new" weapon also raises questions regarding modern forms of combat. The multiple uses of abduction and the relation to the enemy that it expresses may explain why it became such a widespread practice in a highly unorthodox armed conflict.

Key words: Lebanon, civil war, abduction, war violence, captivity.

\section{Introduction}

Nos recherches sur la guerre du Liban de 1975-1990 nous ont amenée à constater la place centrale tenue par une violence qui n'avait jusqu'à présent pas été identifiée comme appartenant à l'univers guerrier : celle des enlèvements. Cette forme originale de la contrainte physique par laquelle un individu capture et retient un corps est un objet historique complexe dont l'étude peine encore à émerger dans le champ de la recherche. À l'exception de certains travaux sur la guerre civile du Mozambique (Legrand, 1995), les productions scientifiques sur le sujet laissent entrevoir les difficultés qui existent à penser cette violence dans sa globalité. Les enlèvements sont en effet étudiés dans le cadre des conflits armés à travers la question des prisonniers de guerre et des détenus des camps tandis qu'ils se formalisent en temps de paix autour des cas de disparitions et de prises d'otages. Dans le premier cas, celui des captivités de guerre, la violence est entendue comme une conséquence des stratégies de rétention des effectifs de l'ennemi et des politiques d'exclusion, d'isolement ou de mise à mort de ces derniers. Dans l'étude des conflits classiques, le captif est un prisonnier, laissant ainsi peu de place à la conceptualisation de captivités informelles qui tendent pourtant à se faire la part belle dans certaines guerres modernes atypiques. Les enlèvements en temps de paix (ou de troubles socio-politiques) ont acquis quant à eux une visibilité dans les sphères politique et académique à travers la formalisation des problèmes liés aux disparitions forcées pratiquées par les régimes autoritaires et aux kidnappings. 
Dans les deux cas, les grilles d'analyse développées agissent comme autant d'écrans qui peinent à rendre compte de l'ensemble des formes et des logiques que les enlèvements peuvent endosser, notamment lorsqu'ils sont massivement mobilisés dans certains conflits aux configurations peu classiques.

Le cas de la guerre civile libanaise (1975-1990) est à ce titre frappant. Bien qu'ayant constitué une des principales façons de combattre au Liban, les dizaines de milliers d'enlèvements restent aujourd'hui dans le silence. Conséquences de la force des écrans évoqués, seuls les cas des captifs disparus ${ }^{1}$ et ceux des otages occidentaux du milieu des années $80^{2}$ font aujourd'hui l'objet d'investigations sérieuses. Le sort des enlevés Libanais et arabes (Palestiniens et Syriens principalement) ayant été échangés, restitués ou tués (et dont les corps ont été retrouvés) restent aujourd'hui dans le silence. Cet état de la recherche témoigne de la difficulté à produire une réflexion globale sur une violence complexe qui reste pourtant l'un des traumatismes de guerre les plus durables pour la population libanaise.

Véritables armes de guerre, les enlèvements au Liban sont un instrument central du répertoire d'action combattant. Leur utilisation massive et leurs logiques protéiformes méritent d'être étudiées car elles permettent d'accéder au plus près du terrain à des mécanismes du conflit encore peu visibles dans un champ de recherche où priment les analyses politiques et géostratégiques. Une telle recherche implique différents niveaux de questionnements. Mise en perspective avec l'ensemble du système guerrier, ses temps et son espace, l'étude des enlèvements interroge les modalités et les stratégies du combat. Chercher à comprendre pourquoi et comment on a enlevé au Liban permet d'interroger la nature des pratiques de violence et apporte un éclairage nouveau sur la structure des antagonismes et les logiques des tensions ayant rythmé la scène libanaise de 1975 à 1990. En questionnant la forme même de la captivité, le chercheur peut également espérer en apprendre davantage sur les expériences de guerre et les vécus traumatiques des victimes, encore laissés à la marge de l'histoire de la guerre qui s'écrit aujourd'hui ${ }^{3}$.

\footnotetext{
${ }^{1}$ Grâce à l'importante mobilisation de la société civile sur la question, il existe aujourd'hui une littérature conséquente sur les disparus, citons ici : Centre Libanais des Droits Humains (CLDH), Rapport: le Liban. Disparitions forcées et détentions au secret, Beyrouth, 21 février 2008 ; Humaydan, S. (2006) : Neither here nor there : families of the disappeared in Lebanon. Master of Art in Social and Behavioural Sciences. American University of Beirut.

${ }^{2}$ Beaucoup d'otages occidentaux ont écrit des ouvrages pour relater leur expérience. Concernant la littérature scientifique notons, Lemasson, S., «La libération des otages allemands au Liban : analyse politique et judiciaire (Partie 1)», Cultures \& Conflits (en ligne), http://conflits.revues.org/index145.html.

${ }^{3}$ Il existe encore très peu de travaux sur les expériences de guerre au Liban. Notons l'initiative en cours Badna Mnaarif (Nous voulons savoir), fruit de la collaboration entre l'Université Saint Joseph et différentes ONG.
} 
Cet article qui s'inscrit dans une recherche en cours se donne pour objectif d'esquisser les premiers éléments d'une anatomie des enlèvements en montrant que la pratique ne se trouve pas à la marge de la guerre mais bien en son plein centre.

\section{Les enlèvements : émergence et centralité de la pratique}

Bien qu'encore non quantifiables, les enlèvements sont une des principales formes adoptées par les violences de guerre au Liban de 1975 à 1990. La pratique des enlèvements existait déjà avant $1975^{4}$, mais le basculement dans la guerre et la violence de grande intensité après le 13 avril 1975 donnent une toute nouvelle dimension à cette pratique. Ce conflit, où les affrontements directs entre groupes armés ne constituaient pas la seule façon de combattre, a vu se généraliser quatre types de violences originales qui ont rythmé le quotidien des combattants et des civils : les tirs des snipers, les bombardements, les explosions (voitures piégées et plasticages) et, enfin, les enlèvements. Le recours à la capture est un phénomène des premiers temps de la guerre et touche indistinctement civils et militaires.

\subsection{Spontanéité et institutionnalisation de la violence}

Dès les premiers jours du conflit, on voit se multiplier enlèvements et contreenlèvements. La configuration de ces violences des origines exprime la spontanéité des premiers affrontements et leur désorganisation profonde dans un contexte où les groupes miliciens ne sont pas stabilisés. Les premiers «enleveurs » sont en majorité des combattants, mais ils se caractérisent par un engagement volontaire, non rémunéré et souvent opéré sur une base locale, notamment celle du quartier. Leurs captures, qui échappent pour partie aux hiérarchies militaires en construction, prennent la forme de réactions à des événements vécus comme des agressions. Il arrive ainsi régulièrement qu'à la suite de l'enlèvement d'un des leurs, les habitants d'un quartier, d'un village ou des particuliers prennent en otage des individus pour négocier un échange. Certaines détentions, opérées au domicile même de l'enleveur, témoignent autant du climat anarchique des débuts de la guerre que de l'imbrication entre vie civile et militaire.

Avec l'institutionnalisation progressive de l'état de guerre et la professionnalisation des combattants, le champ militaire s'autonomise et les captures spontanées tendent à se raréfier pour laisser place à des enlèvements rationalisés et intégrés dans

\footnotetext{
${ }^{4}$ Nous avons pu en recenser durant les événements de 1958, 1969 ou en encore ceux de 1973 (en avril 1973 par exemple, l'enlèvement de deux militaires libanais par le Front Populaire de Libération de la Palestine provoque des affrontements).
} 
les chaînes de commandement des milices. La pratique, massivement mobilisée durant les deux premières années de la guerre (1975-1976), reste une des armes centrales du répertoire d'action combattant et se niche au cœur des stratégies mises en œuvre durant les quinze années de conflit. Des plateformes de discussion et de tractation plus ou moins formelles entre dirigeants militaires, politiques et religieux se mettent en place et la question des enlevés se retrouve souvent au centre des négociations politiques.

\subsection{L’impossible neutralité civile}

Si l'enlèvement touche majoritairement les hommes en âge de combattre, les victimes peuvent être aussi bien civiles que militaires. Les risques d'y être exposé dépendent non pas du degré d'implication effective dans les combats, mais plutôt du degré de menace potentielle que représente l'individu. Le ciblage des victimes se fait donc en fonction de l'évaluation par les combattants de cette même menace, à travers l'identification de certaines caractéristiques désignant l'individu comme ennemi. À travers nos recherches sur les modalités de ce ciblage, nous avons pu constater qu'au même titre que les francs-tireurs et les bombardements, l'enlèvement est une arme qui touche massivement la population civile. Dans un pays où «l'innocence » n'existe plus, chaque individu se voit imposer une identité qui le prive de toute possibilité de neutralité. Chaque homme (et parfois certaines femmes) en âge de combattre peut être envisagé comme suspect et subir une série de tests identitaires destinés à établir sa collusion avec l'ennemi. Une confession, un nom, une provenance géographique ou même un simple aspect physique suffisent à déterminer l'appartenance et à condamner l'individu à devenir une incarnation de l'Autre menaçant. Les identités ont ainsi été essentialisées tout au long d'une guerre qui a prêté aux Libanais des liens plus fantasmés que réels avec les parties prenantes aux combats. Ainsi un chrétien sera-t-il par exemple assimilé à un partisan des Forces Libanaises, alors que 30\% des membres du Parti Communiste Libanais (appartenant en 1975 au bloc «islamo-progressiste », donc ennemi des F.L.) étaient chrétiens. Des lignes de clivages s'imposent donc à tous les membres de la société libanaise, dès lors menacés à travers des identités communautaires et politiques dans lesquelles ils ne se reconnaissaient pas nécessairement.

\subsection{Volatilité des clivages}

De surcroît, une des principales caractéristiques du conflit libanais est la reconfiguration permanente des couples d'antagonistes. Si les débuts de la guerre se structurent autour du clivage entre nationalistes chrétiens et «islamo-progressistes », ce dernier, malgré certaines permanences, se reconfigure rapidement sous l'impulsion de facteurs divers : évolution de la question israélo-arabe, interventions syrienne et 
israélienne, désagrégations internes... Au rythme de ces évolutions, la figure de l'ennemi se redessine et désigne de nouveaux candidats à l'enlèvement au sein de chaque camp. Dans un contexte où les amis d'hier deviennent facilement les ennemis de demain, les civils sont perpétuellement exposés à de nouveaux risques qu'ils peuvent difficilement anticiper. Dans ce contexte, on comprend que les enlèvements intra-communautaires se développent rapidement. À titre d'exemple, après la rupture entre les partisans de Frangié et les Kataëb ${ }^{5}$ auparavant alliés, entre 500 et 1000 personnes sont enlevées dans le Nord Liban les 8 et 9 octobre 1979.

\section{Une violence de guerre massive}

L'enlèvement a été une arme centrale du répertoire d'action combattant. Bien qu'elle ne soit pas mobilisée avec la même intensité tout au long du conflit, elle se caractérise par sa permanence et par l'importance de sa pénétration dans la société civile. Cette forme originale de la contrainte physique apparaît également comme une pratique adaptée à des temporalités et des rythmes guerriers très spécifiques.

\subsection{Permanence de la violence, intermittence de la guerre}

L'enlèvement est utilisé de façon permanente durant les quinze années de conflit, notamment durant les périodes de «crises fuyantes» (Kassir, 1994) relativement calmes. Cette mise en œuvre continue de la pratique des captures s'explique par la configuration originale des violences au Liban. À l'exception de la période 19751976 (dite Guerre des Deux Ans) et de l'invasion israélienne de 1982, les offensives sont relativement rares durant le conflit libanais, et les logiques de conquête et de guerre «totale » quasiment absentes. Les combattants ne cherchent pas à remettre en cause l'existence de l'Autre ni à s'imposer à lui, mais plutôt à s'en séparer, à le contrôler et à confirmer leur hégémonie sur un espace donné. Les stratégies militaires s'adaptent donc aux logiques d'une guerre de position et privilégient les moyens permettant d'épouser les rythmes saccadés et fragmentaires des violences. Le conflit libanais se caractérise ainsi par l'alternance de périodes de calme et de fortes tensions, l'intermittence des combats rendant inefficace l'utilisation des techniques de guerre classiques. Dans ce contexte, les enlèvements permettent de faire la démonstration d'un pouvoir militaire agissant tout en minimisant les coûts consentis. En maintenant un état de guerre minimal, les enlèvements garantissent la perpétuation des tensions et l'impossibilité d'une cessation des hostilités, légitimant ainsi l'existence de l'ordre milicien. Adaptée aux contraintes de l'irrégularité des com-

\footnotetext{
${ }^{5}$ Rupture rendue définitive après le massacre d'Edhen le 13 juin 1978 et l'assassinat par des partisans Kataëb du fils de l'ancien président Frangié.
} 
bats, cette arme est celle d'une guerre paradoxale où l'on évite d'engager des affrontements directs tout en s'employant à maintenir une violence de basse intensité.

\subsection{Omniprésence dans l'espace}

Une géographie de la répartition des enlèvements sur le territoire libanais est encore impossible à établir aujourd'hui avec précision. Les captures eurent lieu principalement à proximité des lignes de front, des axes de circulation, des zones stratégiques (quartiers généraux, permanences de partis) et des espaces où existait une mixité identitaire. Si certaines zones à risque perdurent tout au long de la guerre (frontières Beyrouth Est/Ouest, Zghorta/Tripoli, Sud Liban...), une des caractéristiques du danger est qu'il peut advenir partout et de façon imprévisible. Les Libanais sont majoritairement enlevés lors de contrôles d'identité effectués aux barrages tenus par les miliciens, qui peuvent être fixes ou volants. Les barrages volants sont d'autant plus dangereux pour les civils qu'ils sont imprévisibles et peuvent survenir dans des espaces vécus comme sécurisés. Les enlèvements qui y ont lieu dépendent de la configuration des tensions, des ordres reçus (enlever x... druzes, maronites, etc.) et de l'humeur des miliciens. De nombreuses captures sont également effectuées dans la rue, où des combattants dans une voiture arrêtent puis contrôlent un civil qu'ils décident d'enlever. À partir de 1982, les enlèvements ciblés à domicile se multiplient, menaçant les Libanais jusque dans leur intimité domestique.

Cette omniprésence spatiale des enlèvements peut s'expliquer par les avantages stratégiques que présente cette arme et son adaptation aux contraintes du terrain. La densité du tissu urbain, notamment beyrouthin, rendait les manœuvres d'artillerie lourde difficiles, au profit de modes de combat légers et souples. Les enlèvements fonctionnent en système avec les francs-tireurs et les bombardements pour assurer un contrôle vertical et horizontal de la rue. À ce titre, les captures constituent l'arme idéale pour affirmer physiquement un pouvoir militaire dans des interstices urbains. De façon plus générale et dans un contexte d'extrême fragmentation territoriale, l'objectif militaire central est de contrôler les points stratégiques, les voies de communication et l'espace public. Les enlèvements permettent de traduire physiquement et symboliquement ce contrôle, alors que les milices n'en ont pas nécessairement les moyens logistiques et militaires. Cette omniprésence spatiale des captures et la façon aléatoire dont elles parasitent l'espace public et privé en font une des violences les plus anxiogènes du conflit libanais.

\subsection{Enjeux de la violence faite aux corps}

Au-delà des façons dont elles imprègnent le quotidien des populations et des combattants, les captures constituent une expérience de guerre inédite et traumatique dont les enjeux méritent d'être étudiés. Car les civils ont vécu dans la peur constante 
de l'enlèvement. Contrairement aux combats, aux bombardements et aux francstireurs, l'enlèvement ne peut être anticipé et les stratégies d'accommodation développées par les civils sont précaires. Si des parcours d'abris protégeant des francstireurs ont été mis en place dans les rues, comment en effet échapper à un barrage volant?

Les captures présentent des degrés variables de violence, mais la plupart se produisent dans un climat de grande tension et de brutalité. Le civil désarmé subit, parfois devant ses proches, un contrôle humiliant et effrayant sous la pression des miliciens dont il attend le verdict. Ces derniers inspectent ses papiers, son allure et même sa façon de parler. L'entrée en captivité à proprement parler s'accompagne souvent de brimades et d'insultes, le civil étant généralement amené vers un véhicule destiné à le transporter jusqu'à son lieu de détention. Dans la majorité des cas, l'enlevé fait l'expérience de son voyage dans le noir, soit qu'il ait été projeté dans le coffre de la voiture, soit qu'il ait été encagoulé. Bien que nos enquêtes nous aient montré que cette cécité était paradoxalement préférable (un homme qui voit encore est souvent un homme que les miliciens ont déjà décidé de tuer...), on comprend aisément la terreur d'un individu embarqué vers une destination inconnue et ne pouvant apprivoiser la menace par la vue. Le captif sait par ailleurs qu'il n'existe que quatre trajectoires possibles à son enlèvement : l'échange, la restitution "gratuite », la mort, la disparition. Les tortures, très répandues en captivité et dont les récits de captifs restitués ou retrouvés mutilés témoignent, deviennent la principale source de terreur de la population, notamment pendant les deux premières années de la guerre.

L'enlèvement est une forme inédite de la violence de guerre dont la nature même implique des enjeux symboliques et des traumatismes pour les victimes encore peu reconnus aujourd'hui. Bien que brutaux, les tirs, les bombardements et les coups ont une issue immédiate: le choc, la mort, la blessure. En revanche, l'issue de l'enlèvement n'est pas immédiate; sa violence s'inscrit dans des temps longs (de quelques heures à des dizaines d'années), marqués du sceau de l'incertitude. Incapable d'interférer sur le déroulement de sa captivité, la victime évolue dans un environnement hostile qu'elle ne maitrise pas et dont l'issue, différée, peut lui être fatale à n'importe quel instant. L'enlevé n'étant pas un prisonnier officiel, il vit en suspension, hors de l'espace social et subit son expérience comme une parenthèse anxiogène sur laquelle il n'a aucun contrôle. Certains combattants ont également fait volontairement disparaître des captifs alors privés de toute trace de leur propre mort ${ }^{6}$. Le problème des disparus est ainsi un enjeu mémoriel central au Liban depuis 1990. Car rendre invisible la mort revient à nier l'existence même, et les disparitions ont

\footnotetext{
${ }^{6}$ « La pure et simple disparition est la négation même de l'individu, comme s'il n'avait jamais existé » Françoise Héritier, De la Violence, tome 1, Paris, Odile Jacob, mars 2005, p.16.
} 
laissé des milliers de familles, privées de toute sépulture, dans l'impossibilité de faire leur deuil.

\section{Une arme aux logiques protéiformes}

Comprendre pourquoi les enlèvements ont été si massivement mobilisés pendant le conflit libanais implique de s'interroger sur les logiques qui sous-tendent l'acte de violence.

\section{1. Économie de troc et mécanismes de vengeance}

La majorité des enlèvements au Liban ont été pratiqués à l'aveugle, c'est-à-dire sur une cible inconnue et choisie au hasard, en fonction d'une appartenance à des critères retenus comme menaçants à un moment donné (religion, nom, allure...). Ces captures aveugles pouvaient viser régulièrement deux objectifs : l'échange et/ou la vengeance.

Dès les premiers jours du conflit, les enlèvements et contre-enlèvements se succèdent. Souvent motivés par la perte d'un proche ou d'un camarade lui-même capturé, ces enlèvements sont le moyen de pression utilisé pour exiger une restitution. Pratiquées de façon spontanée et massive au début du conflit, ces captures tendent à se rationaliser avec la professionnalisation des milices. Le troc devient ainsi une véritable façon de combattre et l'instrument grâce auquel les combattants négocient un espace, un rapport de force, des ressources. Les miliciens, sur ordre de leur hiérarchie, capturent X... membres d'une communauté, qu'ensuite ils relâchent/tuent/font disparaître en fonction des négociations et des événements. Les victimes deviennent de simples monnaies d'échange et l'instrument par lequel se négocie l'allocation des ressources en temps de guerre.

Dès les premiers temps de la guerre, les enlèvements à l'aveugle sont également motivés par des logiques de vengeance. Ces captures visent à punir l'Autre de la douleur qu'il «nous » a infligée et à lui rendre le coup porté. Elles prennent pour cible des civils innocents, l'individu étant tenu responsable des agissements de la communauté à laquelle il est censé appartenir. Ces rituels de vengeance peuvent être individuels ou collectifs. Dans le premier cas, un homme perd un proche et décide de punir un ou des boucs émissaires en les enlevant. Un individu innocent, mais qui partage avec le coupable du crime certains signes d'appartenance (religion, parti politique, origine géographique...), sera alors capturé, comme si châtier l'innocent revenait à punir le coupable par procuration. Le 7 septembre 1975 par exemple, un jeune zghortiste tue douze otages quand il apprend la mort de son frère lors d'affrontements à Kobbé (Tripoli). Dans le cas des vengeances collectives, des enlèvements-vendettas viennent châtier le groupe ennemi qui a porté atteinte symboliquement et collectivement à son propre groupe. Ainsi de nombreuses captures sui- 
vent-elles les assassinats des chefs charismatiques Kamal Joumblatt, leader du MNL tué en mars 1977, et Bachir Gemayel, leader des Forces Libanaises tué en septembre $1982^{7}$. Ces logiques de représailles perdurent tout au long du conflit et sont un des principaux moteurs de la violence de l'enlèvement.

\subsection{L'arme d'une guerre territoriale}

Enlever signifie littéralement retirer un corps d'un espace pour le déplacer dans un autre. Cette démarche d'extraire les corps, d'éliminer leur présence physique d'un espace, nous a amené à questionner le rapport entre les formes prises par la violence et «l'obsession territoriale ${ }^{8}$ des combattants. Au Liban, la pratique de l'enlèvement est productrice de frontières physiques et symboliques qu'il faut envisager autour de quatre dynamiques : homogénéiser, protéger, purifier, contrôler.

Dans un pays où le voisin est devenu ennemi et où l'espace est partagé, les actions militaires cherchent à infléchir et redéfinir la répartition des individus en fonction de frontières construites entre soi et l'Autre. Dès le début du conflit, les combattants affichent leur volonté d'homogénéiser les espaces autour d'identités vécues comme sécurisantes. Les enlèvements deviennent alors l'instrument d'une ségrégation spatiale qui vise à éliminer la présence de l'Autre et à transformer la composition sociale des quartiers en obligeant la population à se replier sur des micro-territoires homogènes. En enlevant, on extrait symboliquement et physiquement l'Autre de soi et on bannit les possibilités d'une coexistence. Ces captures prennent parfois des dimensions de véritable rituel purificateur où l'Autre est perçu comme celui qui souille l'espace sacré de la communauté. La pratique du Sahl («traîner » en arabe) est à cet égard intéressante puisqu'elle maximise l'effraction symbolique faite à l'adversaire : une fois capturées, les victimes sont accrochées à l'arrière des voitures et traînées pour réaffirmer par le sang les frontières d'un espace qui est celui de l'entre-soi. Une fois les territoires ainsi «sécurisés », les combattants pratiquent de nombreux enlèvements destinés à en protéger les frontières en installant des barrages bloquant et contrôlant les voies d'accès. Les barrages, décrits par Fawaz Traboulsi comme les «avant-postes du hima» (enclos), inscrivent ainsi dans l'espace les ségrégations symboliques tout en empêchant durablement l'Autre d'y pénétrer.

Le rapport des combattants à leur territoire est cependant ambigu et l'enlèvement apparaît parfois comme l'instrument trivial d'une domination spatiale à la limite de la gestion mafieuse. Dans un contexte de désagrégation de la puissance publique et

\footnotetext{
${ }^{7}$ En 1977, des enlèvements ont eu lieu dans le Chouf (région dont est originaire K. Joumblatt) tandis qu'en 1982, des rafles massives ont eu lieu à Beyrouth Ouest pour venger la mort de B. Gemayel.

${ }^{8}$ Picard, E. (1996), «Liban, matrice historique », Jean, F., Rufin, J.C. (dirs), Économie des guerres civiles, Paris : Hachette, collection pluriel, p.89.
} 
d'appropriation des ressources par les groupes miliciens, les captures servent aussi à contrôler les flux de biens et de personnes. En contraignant l'utilisation de l'espace et en y affirmant une mainmise militaire, les combattants réussissent à s'approprier ou à contester certains monopoles de la puissance publique ${ }^{9}$ et à devenir les autorités de facto des territoires. Les multiples enlèvements de camions-citernes et de leurs occupants sur l'autoroute Tripoli-Beyrouth traduisent par exemple l'objectif milicien d'appropriation d'une manne économique et son utilisation comme moyen de pression sur les populations de la capitale sans que l'État ne puisse réellement intervenir. Ces types de captures permettent de restructurer les espaces et de canaliser le flux de ressources pour qu'ils profitent aux groupes armés. En observant les pratiques des enlèvements, on constate que les logiques de purification et les motivations plus prosaïques de domination territoriale ne s'excluent pas nécessairement. Il semble cependant que les logiques visant la perpétuation du pouvoir milicien aient prévalu sur la période allant de 1983 à 1990. Le conflit, alors structuré par le repli sur des micro-territoires identitaires cloisonnés et par la multiplication des conflits intracommunautaires, voit se répandre les enlèvements-rackets et les captures crapuleuses.

\subsection{Ambiguïtés des rapports à l'ennemi}

Au regard de l'originalité de notre objet en termes de mode d'infliction de la violence, nous avons été amenée à formuler des questions qui restent aujourd'hui encore sans réponses définitives. Que devons-nous comprendre de la volonté des combattants d'extraire et de retenir les corps ? Que nous apprend cet acte du rapport à l'ennemi et des matrices de violence au Liban?

Dans un pays où l'objectif des combats n'était pas l'anéantissement définitif de l'Autre et où la figure de l'ennemi n'était pas stabilisée, l'enlèvement semble exprimer l'ambiguïté du rapport à l'adversaire. Si des massacres ont bien eu lieu ponctuellement au Liban, les exactions contre la population prenaient une forme diffuse témoignant de la généralisation du sentiment de méfiance plus que d'une haine stable et durable. L'enlèvement est une violence dont les issues peuvent être multiples. En enlevant, non seulement le combattant se permet de déplacer les corps pour les éliminer loin des yeux de la population, mais il diffère également le moment de sa prise de décision quant au sort du captif. Dans une société «brutalisée » où les violences perdurent, les différents groupes combattants redessinent constamment la figure de leur adversaire et désignent parmi leurs alliés de nouvelles menaces, plus intimes. Cette fragmentation politique et la très grande volatilité des an-

\footnotetext{
${ }^{9}$ Comme la diffusion télévisée (TéléLiban), la poste, la police ou encore le port de Beyrouth par exemple.
} 
tagonismes s'expliquent peut-être par le besoin d'une société de désigner de nouveaux responsables à des tensions qui subsistent et qu'elle n'arrive pas à résorber. À ce titre, les comportements paranoïaques se généralisent et chacun cherche à redéfinir la pureté de son groupe en excluant ceux qu'il estime représenter une nouvelle menace. À ce titre, ne peut-on pas comprendre le choix de l'enlèvement comme l'acte d'extraire de l'entre-soi un individu rendu momentanément suspect sans qu'il y ait pour autant de volonté de s'assurer de son anéantissement total ?

\section{Conclusion}

Les enlèvements au Liban constituent un objet historique complexe, autant pour des raisons relevant de nos difficultés à nous départir de certains présupposés que du fait des limites méthodologiques et matérielles de nos enquêtes. Les principales difficultés rencontrées tiennent à l'absence de source sur le sujet et à la focalisation de toutes les études sur les cas des prises d'otages d'Occidentaux et sur les disparus. Actuellement non quantifiables, les enlèvements sont une violence de guerre sur laquelle il est difficile d'obtenir des informations dans un contexte d'absence de renouvellement de la classe politique depuis la guerre et de résurgences régulières des tensions. Cette enquête peut et doit malgré tout être menée. L'enlèvement constitue un des traumatismes les plus durables de la guerre de 1975-1990. Il est frappant de constater qu'à l'hypermnésie de cette violence dans les mémoires familiales et individuelles répond le silence dans les discours politiques et les productions savantes. À ce titre, il semble important d'approfondir les études permettant de rendre visible cette expérience de guerre massivement vécue par les civils et les combattants, et qui reste une des grandes oubliées de l'histoire libanaise. Reconnaître que les captures ont constitué une arme centrale du conflit nous oblige également à repenser les formes classiques du combat. Dans une guerre complexe où s'imbriquent les logiques internes et externes, où l'ennemi est en perpétuelle redéfinition et où les objectifs des combattants sont difficiles à saisir, il est indispensable d'être attentif aux formes originales que peuvent prendre les violences. Au Liban, l'enlèvement s'est imposé comme l'arme la mieux adaptée pour répondre aux enjeux de la lutte sur le terrain, jusqu'à devenir un instrument central du répertoire d'action combattant. L'étude de cette arme longtemps ignorée permet de dévoiler des logiques de guerre inédites ainsi que d'appréhender les significations multiples qui ont sous-tendu les actes de violence. Le retrait et la capture massive des corps au Liban révèlent une guerre discontinue, liminale où les enjeux de territorialité sont centraux et les moyens logistiques de contrôle des espaces assez faibles. L'enlèvement a constitué une arme protéiforme mobilisée pour atteindre des objectifs extrêmement divers : la vengeance, des ressources économiques, politiques, militaires, le contrôle d'un espace, ou plus simplement encore la terreur des populations. Son usage massif au Li- 
ban en fait encore aujourd'hui une arme incontournable en période de tension ${ }^{10}$ témoignant de son imprégnation profonde dans le corps social et le langage politique.

$\mathrm{Au}$ regard de nos recherches actuelles, nous pouvons affirmer que le Liban n'est pas le seul pays où les enlèvements ont envahi le fait guerrier. Au Mozambique durant la guerre de 1976 à 1992, des centaines de milliers de personnes ont été ainsi enlevées. La capture correspond à des terrains où les mécanismes guerriers sont ambigus, intriqués et complexes. La souplesse de ce moyen, la multiplicité de ses usages possibles et les rapports équivoques à l'ennemi qu'il dessine, expliquent peut-être que l'enlèvement tende à se frayer une place importante dans les conflits peu classiques. Quoi qu'il en soit, il semble aujourd'hui indispensable, au regard de l'importance du nombre de victimes, de reconnaître dans l'enlèvement une des formes atypiques mais redoutables que peut adopter la violence de guerre.

\section{Bibliographie}

AudoIn-RouzeAu, S. (2008). Combattre. Une anthropologie historique de la guerre moderne (XIX ${ }^{e}-X X I^{e}$ siècle), Paris : Seuil.

BEydoun, A. (1993). Itinéraires d'une guerre incivile, Paris : Karthala-CERMOC.

Centre Libanais des Droits Humains (CLDH). Rapport: le Liban. Disparitions forcées et détentions au secret, Beyrouth, 21 février 2008.

DAVIE, M. (1983). «Comment fait-on la guerre à Beyrouth ? », Hérodote, 29/30, pp.17-56.

HuMAYDAN, S. (2006). Neither here nor there: families of the disappeared in Lebanon. Master of Art in Social and Behavioural Sciences. American University of Beirut.

KASSIR, S. (1994). La guerre du Liban. De la dissension nationale au conflit régional (1975-1982), Paris : Karthala.

LEGRAND, J-C, (1995). «Passé et présent dans la guerre du Mozambique: les enlèvements pratiqués par RENAMO », Lusotopie, 3/4.

LABAKI, B. et ABOU-RJEILI, K. (1993). Le bilan des guerres libanaises 1975-1990, Paris : L'Harmattan, 1993.

\footnotetext{
${ }^{10} \mathrm{Il}$ y a eu des cas d'enlèvements à Beyrouth Ouest en 2008 lors du coup de force du Hezbollah, mais l'exemple le plus frappant concernant les possibilités de résurgence de la pratique est surement la multiplication des enlèvements (notamment dans la Békaa) qui a lieu depuis début 2013 et le débordement (officiel) du conflit syrien au Liban.
} 
NAHOUM-GRAPPE, V. (2002). «Anthropologie de la violence extrême : le crime de profanation », Revue internationale des sciences sociales, 174, pp.601-609.

NASSIF TAR KOVACS, F. (1998). Les rumeurs dans la guerre: Liban 1975-1977, les mots de la violence, Paris : CNRS-Éditions.

PICARD, E. (1998). Liban. État de discorde, des fondations aux guerres fratricides, Paris : Flammarion.

PiCAUdou, N. (1989). La déchirure libanaise, Bruxelles : Édition Complexes.

TRABOULSI, F. (1993). Identités et solidarité croisées dans les conflits du Liban contemporain, Thèse de doctorat en histoire, Université Paris VIII. 